\title{
Modeling the Potential Impact of the 2014 American Academy of Pediatrics Respiratory Syncytial Virus Prophylaxis Guidance on Preterm Infant RSV Outcomes
}

\author{
Kimmie K. McLaurin · Archana Chatterjee $\cdot$ Doris Makari
}

To view enhanced content go to www.infectiousdiseases-open.com

Received: September 16, 2015 / Published online: October 26, 2015

(C) The Author(s) 2015. This article is published with open access at Springerlink.com

\section{ABSTRACT}

Introduction: The American Academy of Pediatrics (AAP) Committee on Infectious Diseases issued updated guidance on respiratory syncytial virus (RSV) prophylaxis in 2014. This report models the potential impact of the new guidance on RSV outcomes in preterm infants 29-34 weeks' gestational age (wGA) without chronic lung disease in the United States.

Methods: The number of preterm infants was estimated using 2012 natality data. Palivizumab utilization prior to the 2014 guidance update was estimated using 2013-2014 specialty pharmacy utilization data. Low, moderate, and high RSV hospitalization (RSVH) rates as well as average hospital length of stay, intensive care unit (ICU) admissions and mechanical

Electronic supplementary material The online version of this article (doi:10.1007/s40121-015-0097-3) contains supplementary material, which is available to authorized users.

K. K. McLaurin $(\varangle) \cdot$ D. Makari

AstraZeneca, Gaithersburg, MD, USA

e-mail: Kimmie.McLaurin@astrazeneca.com

A. Chatterjee

University of South Dakota Sanford School of

Medicine, Sioux Falls, SD, USA ventilation (MV) frequencies were derived from published observational studies. Palivizumab efficacy was derived from two randomized clinical trials. RSV events that would be attributable to the 2014 guidance change were calculated for preterm infants 29-31 and 32-34 wGA.

Results: Annual number of infants 29-34 wGA surviving the neonatal period was estimated at 123,687 . Of these, an estimated 44,712 (37\%) would receive palivizumab based on the 2012 guidance. The annual number of RSVH among infants 29-34 wGA would increase from 3580 under the 2012 guidance to 6166 under the 2014 guidance based on moderate rates. This would result in an additional 24,440 hospitalization days, 1162 ICU admissions, and $584 \mathrm{MV}$ events among this population.

Conclusions: Based on published historical and contemporary data on RSVH rates in preterm infants 29-34 wGA, the 2014 AAP guidance is expected to result in additional burden to the healthcare system and families of preterm infants. The impact of the new guidance will be difficult to detect among the overall infant population, particularly in settings without routine testing for RSV, but the impact will be 
substantial for the small high-risk population affected by the changes.

Funding: AstraZeneca.

Keywords: Hospitalization; Modeling;

Palivizumab; Preterm; RSV

\section{INTRODUCTION}

Respiratory syncytial virus (RSV) is the most common cause of acute lower respiratory tract infection and a major cause of hospitalizations in young children [1]. In preterm infants, the risk for severe RSV disease may lead to hospitalization and, in some cases, intensive care unit (ICU) admissions and mechanical ventilatory support [2].

Palivizumab (MedImmune, Gaithersburg, MD, USA) is a monoclonal antibody indicated for the prevention of serious lower respiratory tract disease caused by RSV in high-risk children [3]. The American Academy of Pediatrics (AAP) first published guidance for use of palivizumab prophylaxis against RSV in 1998 and subsequently updated them in 2000, 2003, 2006, 2009, and 2012 [4]. These later iterations of the guidance narrowed the population recommended for prophylaxis with a focus on the increased risk and severity of RSV disease associated with younger gestational age and younger chronologic age [4, 5]. The 2012 Committee on Infectious Diseases (COID) guidance recommended use of palivizumab during the RSV season in preterm infants $\leq 28$ weeks' gestational age (wGA) and $<12$ months of age at RSV season start, 29-31 wGA and $<6$ months of age at RSV season start, and 32-34 wGA and $<3$ months of age at RSV season start. In addition to being $<3$ months of age, infants 32-34 wGA were required to have additional risk factors of either day care and/or school-age siblings and could only receive prophylaxis through 90 days of age [6]. In July 2014, the AAP issued updated guidance on RSV prophylaxis that no longer recommended palivizumab for preterm infants born 29-34 wGA without chronic lung disease (CLD), asserting that "data regarding the risk of RSV hospitalization for most preterm infants do not support a benefit from prophylaxis [4]".

However, there is controversy surrounding the elimination of palivizumab use among infants 29-34 wGA. It has been argued that the recommendation is not justified by the studies cited by the COID [7, 8]. Boyce et al. noted, "as academic physician-scientists, we should carefully evaluate all of the existing data and properly study proposed changes before further restricting palivizumab use in this voiceless and vulnerable population of infants [7]". Similarly, the Institute of Medicine's Standards for Developing Trustworthy Clinical Practice Guidelines recommend that guidelines include descriptions of the potential benefits and harms [9].

The current analysis was designed to address this need to evaluate the potential impact of the AAP guidance change on RSV hospitalizations in US preterm infants. By constructing a model that elucidates the expected RSV outcomes for preterm infants per the 2012 guidance compared with the 2014 guidance, we were able to estimate the potential impact of the 2014 AAP guidance on RSV outcomes in preterm infants born 29-34 wGA without CLD during the 2014-2015 and future RSV seasons.

\section{METHODS}

This article does not contain any new studies with humans or animal subjects performed by 
any of the authors. Eight steps were taken to model the potential impact of the 2014 AAP guidance on RSV outcomes in preterm infants 29-31 wGA and $<6$ months chronologic age at RSV season start and infants 32-34 wGA and $<90$ days chronologic age with $\geq 1$ risk factor (Fig. 1). The first step involved identifying the total number of US preterm births surviving the neonatal period using the 2012 CDC Natality File and Linked Birth/Infant Death Data File [10]. In step 2, the number of preterm infants 29-31 and 32-34 wGA without CLD who would have received palivizumab on the basis of the 2012 AAP guidance were estimated using Specialty Distribution Network outpatient usage data from the 2013-2014 RSV season. For example, infants 32-34 wGA who would have received palivizumab were assumed to be less than 90 days of age during the RSV season and had at least one additional risk factor (daycare attendance or preschool-aged siblings). Step 3 involved selecting low, moderate, and high rates of RSV hospitalization (RSVH) for infants born at 29-31 and 32-34 wGA who would have received palivizumab under the 2012 guidance and for infants 29-31 and 32-34 wGA who would have not received palivizumab under the 2012 guidance. These hospitalization rates were estimated using historical and contemporary data from published studies [11-13]. The low, moderate, and high RSVH rates for infants 29-31 wGA $<6$ months at RSV season start who would have received palivizumab were $6.0 \%, 8.5 \%$, and $12.0 \%$, respectively. Among infants 32-34 wGA $<90$ days of age during the RSV season with one additional risk factor (daycare attendance or preschool-aged siblings), the low, moderate, and high RSVH rates for infants that would have received palivizumab were $5.0 \%, 6.5 \%$, and $10.0 \%$, respectively [11]. Observational studies would suggest that infants $29-31$ wGA and $32-34$ wGA that would have not received palivizumab (those $>6$ months at RSV season start for 29-31 wGA and those >90 days or those $<90$ days during the season without risk factors for infants 32-34 wGA) would have lower RSVH risk than infants of the same wGA group that was identified to receive palivizumab. Therefore, the low, moderate, and high RSVH rates were estimated to be $3.0 \%, 4.5 \%$, and $6.0 \%$ for infants $29-31 \mathrm{wGA}$ and $2.0 \%, 3.5 \%$, and $5.0 \%$ for infants $32-34$ wGA, respectively. From these rates and the known population sizes, the total annual number of RSVH among preterm infants 29-31 and 32-34 were calculated.

Next, the RSVH relative risk reduction rates associated with palivizumab use were applied in step 4 ; a relative risk reduction of $78 \%$ for preterm infants without bronchopulmonary dysplasia was obtained from the IMpact study findings, which are similar to recent results of Blanken et al. [12, 14]. In step 5, the number of hospitalizations with palivizumab use based on the 2012 AAP guidance was calculated, and in step 6, the number of hospitalizations with palivizumab use based on the 2014 AAP guidance was calculated. These estimates were then subtracted to calculate the number of additional hospitalizations expected from the change in guidances (step 7). Finally, in step 8, hospitalization length of stay, ICU rates, and mechanical ventilation rates were applied from an observational study of RSV-confirmed hospitalizations among US preterm infants 29-35 wGA that did not receive palivizumab [15-17]. The average hospitalization length of stay was 10.4 days in infants 29-31 wGA and 8.4 days in infants $32-34$ wGA and $<90$ days chronologic age. The average ICU rates were $48.1 \%$ in infants $29-31$ wGA and $41.4 \%$ in infants $32-34$ wGA and $<90$ days chronologic 
Step 1: Identify total number of US preterm births surviving the neonatal period

(Using 2012 CDC Natality File and Linked Birth/Infant Death Data [10])

Step 2: Estimate number of preterm infants 29-34 wGA without CLD who would have been eligible for and receive palivizumab on the basis of the 2012 AAP guidance

(Using 2013-14 RSV-season specialty pharmacy utilization data)

\begin{tabular}{|l|c|c|c|}
\hline \multirow{2}{*}{ Infant Group } & $\begin{array}{c}\text { Number of } \\
\text { births }\end{array}$ & $\begin{array}{c}\text { Eligible and received } \\
\text { palivizumab }\end{array}$ & $\begin{array}{c}\text { Number of } \\
\text { infants }\end{array}$ \\
\hline \multirow{2}{*}{$29-31$ wGA } & \multirow{2}{*}{29,106} & Yes & 20,474 \\
\cline { 3 - 4 } & \multirow{2}{*}{$32-34$ wGA } & No & 8,632 \\
\hline \multirow{2}{*}{94,581} & Yes & 24,238 \\
\cline { 3 - 4 } & & No & 70,343 \\
\hline
\end{tabular}<smiles></smiles>

Step 3: Provide low, moderate, and high estimates for the RSV hospitalization rates in infants 29-34 wGA who would have received palivizumab based on the 2012 AAP guidance (Using hospitalization rates from previously published studies [12,13])

\begin{tabular}{|l|c|c|c|}
\hline & Low & Moderate & High \\
\hline 29-31 wGA received palivizumab & $6.0 \%$ & $8.5 \%$ & $12.0 \%$ \\
\hline 29-31 wGA did not receive palivizumab & $3.0 \%$ & $4.5 \%$ & $6.0 \%$ \\
\hline 32-34 wGA received palivizumab & $5.0 \%$ & $6.5 \%$ & $10.0 \%$ \\
\hline 32-34 wGA did not receive palivizumab & $2.0 \%$ & $3.5 \%$ & $5.0 \%$ \\
\hline
\end{tabular}

Step 4: Apply RSV hospitalization relative risk-reduction rates from use of palivizumab (Using 78\% obtained from the IMpact study findings [14])

Step 5: Calculate the number of hospitalizations expected on the basis of the 2012 AAP guidance with palivizumab use

Step 6: Calculate the number of hospitalizations expected on the basis of the 2014 AAP guidance without palivizumab use

Step 7: Calculate the number of additional hospitalizations expected from the change in guidances Number of hospitalizations in step 6-number of hospitalizations in step 5

Step 8: Apply estimated average hospitalization length of study, ICU rates, and mechanical ventilation rates (Using data from an observational study of RSV-confirmed hospitalizations [15])

\begin{tabular}{|l|c|c|c|}
\hline & $\begin{array}{c}\text { Hospitalization } \\
\text { Length of Stay }\end{array}$ & ICU Rates & $\begin{array}{c}\text { Mechanical } \\
\text { Ventilation Rates }\end{array}$ \\
\hline $29-31$ wGA & 10.4 & $48.1 \%$ & $25.9 \%$ \\
\hline $32-34$ wGA & 8.4 & $41.4 \%$ & $18.9 \%$ \\
\hline
\end{tabular}


4Fig. 1 Modeling the potential impact of the 2014 AAP guidance on RSV outcomes in preterm infants. AAP American Academy of Pediatrics, $C D C$ Centers for Disease Control and Prevention, CLD chronic lung disease, $I C U$ intensive care unit, $R S V$ respiratory syncytial virus, $w G A$ weeks' gestational age

age. The average mechanical ventilation rates were $25.9 \%$ in infants $29-31$ wGA and $18.9 \%$ in infants $32-34$ wGA and $<90$ days chronologic age.

The model was created using Excel 2013 (Microsoft Corporation).

\section{RESULTS}

The annual number of infants 29-34 wGA surviving the neonatal period was estimated at $123,687(29,106$ infants $29-31$ wGA and 94,581 infants $32-34$ wGA; Fig. 2); this is approximately $3 \%$ of the total annual number of US births, 3,937,686. Of these infants, an estimated 44,712 (37\%) would have received palivizumab based on the 2012 AAP guidance, including 20,474 (70\%) infants 29-31 wGA and 24,238 (26\%) infants 32-34 wGA.

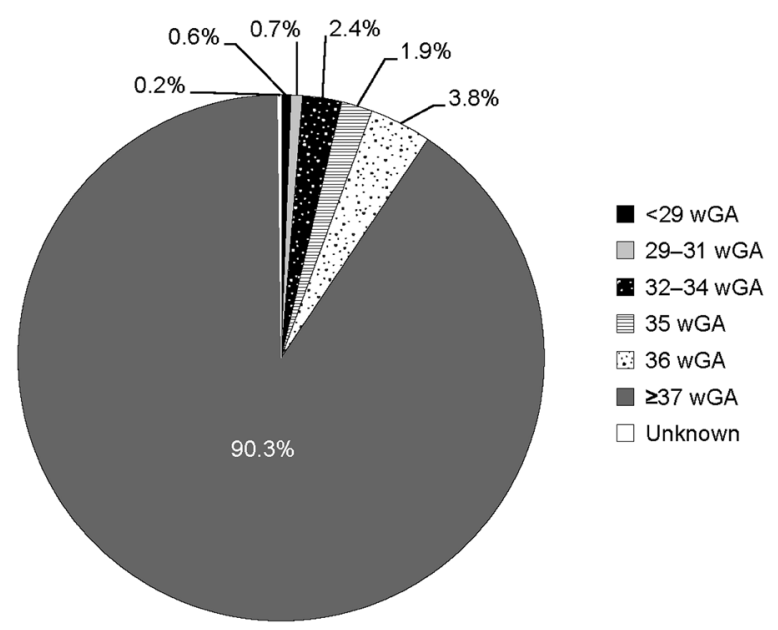

Fig. 2 US births by gestational age after adjusting for neonatal deaths. $w G A$ weeks' gestational age
The low, moderate, and high estimates for the total number of RSVH expected post-2014 guidance in infants 29-31 wGA were 1487, 2129, and 2975, respectively. The low, moderate, and high estimates for the total number of RSVH expected in infants 32-34 wGA were 2619, 4037, and 5941, respectively.

Using low estimates, the number of additional RSVH that would be associated with the 2014 guidance relative to the 2012 guidance was 958 for infants 29-31 wGA and 945 for infants 32-34 wGA (Fig. 3a). Based on moderate estimates, the numbers of additional RSVH that would be associated with the 2014 guidance relative to the 2012 guidance were 1357 and 1229 for infants 29-31 and 32-34 wGA, respectively (Fig. $3 \mathrm{~b}$ ). The high estimates for the number of additional RSVH that would be associated with the 2014 guidance relative to the 2012 guidance were 1916 for infants 29-31 wGA and 1891 for infants 32-34 wGA (Fig. 3c).

The low estimates for the number of additional RSVH that would be associated with the 2014 guidance relative to the 2012 guidance would result in an additional 9965 and 7940 hospitalization days, 461 and 391 ICU admissions, and 248 and 179 mechanical ventilation events, in infants 29-31 wGA and 32-34 wGA, respectively. The moderate estimates for the number of additional RSVH that would be associated with the 2014 guidance relative to the 2012 guidance would result in an additional 14,117 and 10,322 hospitalization days, 653 and 509 ICU admissions, and 352 and 232 mechanical ventilation events, in infants 29-31 wGA and 32-34 wGA, respectively. Using high estimates, this would result in an additional 19,930 and 15,881 hospitalization days, 922 and 783 ICU admissions, and 496 and 357 mechanical ventilation events in infants 29-31 wGA and 32-34 wGA, respectively. 

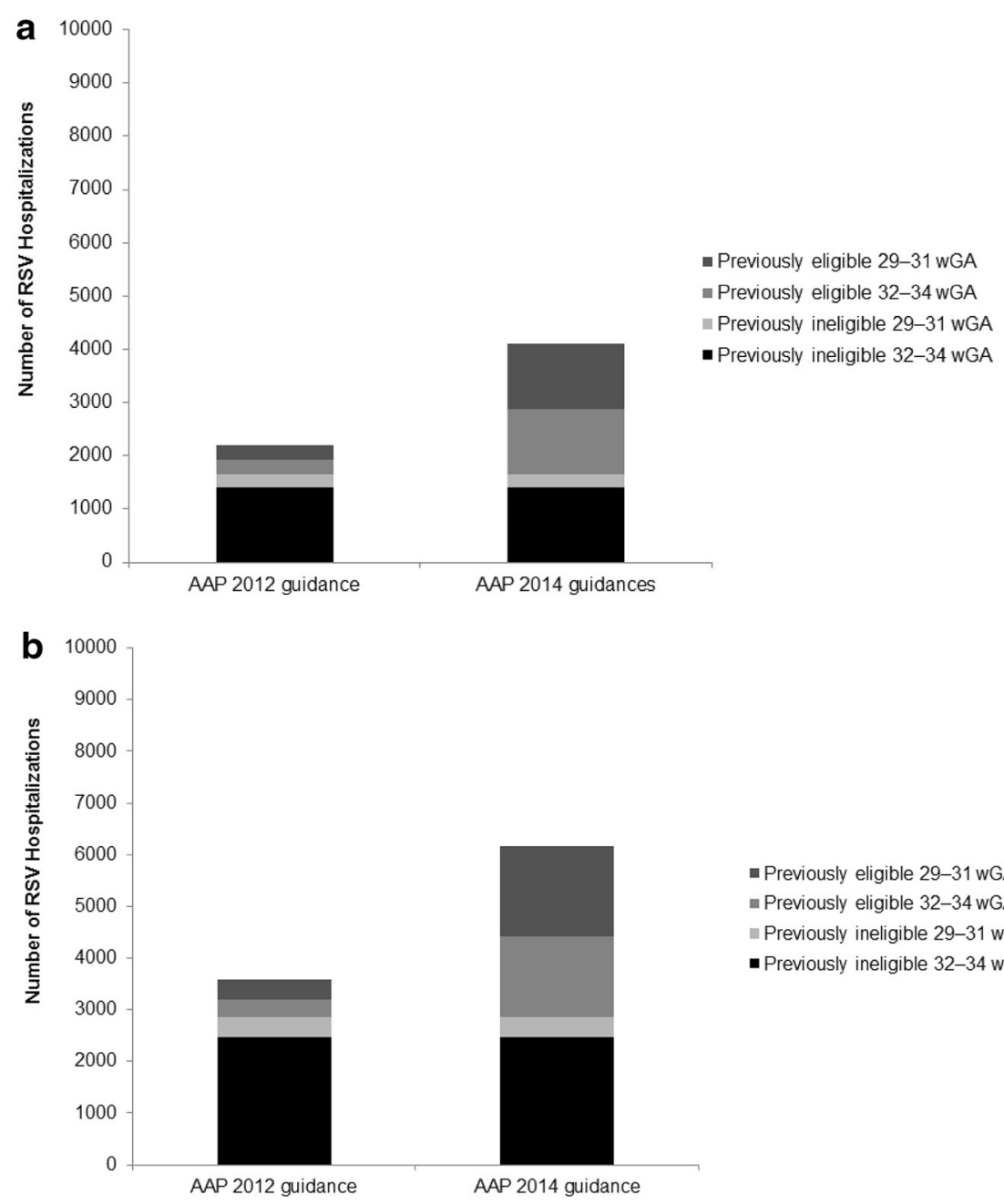

- Previously eligible 29-31 wGA - Previously eligible $32-34$ wGA - Previously ineligible 29-31 wGA - Previously ineligible 32-34 wGA

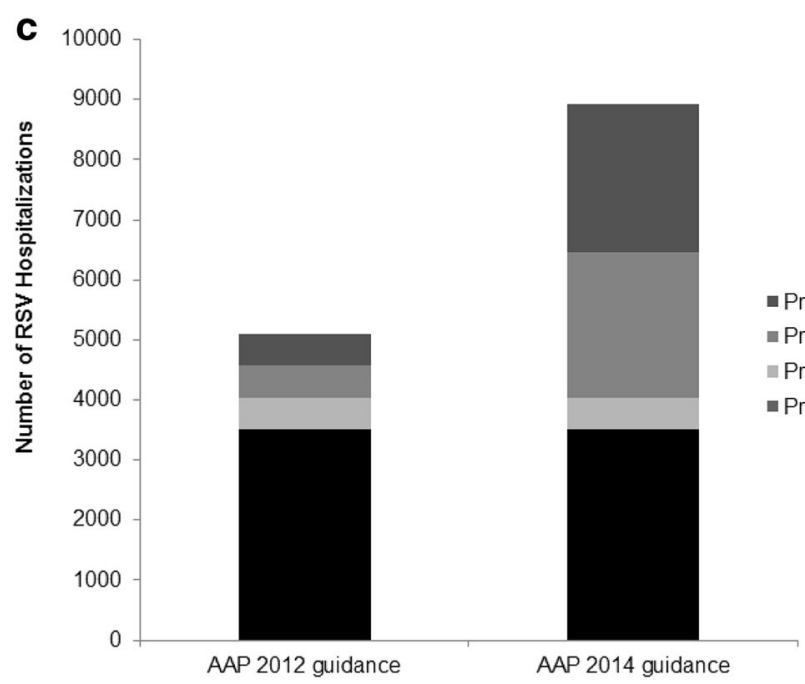

Fig. 3 Estimated number of RSV hospitalizations among infants 29-31 wGA and 32-34 wGA based on a low, $\mathbf{b}$ moderate, and $\mathbf{c}$ high estimates. $A A P$ American Academy of Pediatrics, $R S V$ respiratory syncytial virus, $w G A$ weeks' gestational age 


\section{DISCUSSION}

The current analysis quantifies the size of the population affected by the 2014 policy change and the range of expected additional RSV hospitalizations in that population in the absence of palivizumab use. This quantification of the negative or serious consequences of a new clinical practice guidance is a recommended best practice by the Institute of Medicine [9].

The data justifying the 2014 COID guidance on palivizumab use have been questioned, because they rely on reinterpretation of historical studies that were previously cited by AAP in support of palivizumab use, along with newer studies conducted in years in which palivizumab use was widespread $[7,11]$. The 2014 AAP policy asserted, "In recent large cohort studies of moderately preterm infants, the majority of whom did not receive palivizumab, $2.5 \%$ to $4.9 \%$ required hospitalization for RSV infection during the RSV season indicating that more than $95 \%$ did not require hospitalization [4]". A review of historical and contemporary studies suggests that this range is an underestimate, consistent with the fact that the cited study did not employ active surveillance or active testing for RSV.

Similarly, without active case-finding methods to evaluate the burden of RSV disease among US preterm infants, the overall adverse outcomes and healthcare costs associated with the new 2014 AAP guidance may be easily overlooked due to the small number of infants affected by this guidance. Preterm infants born 29-34 wGA comprise $3.1 \%$ of live births, whereas those born 29-31 wGA represent only $0.7 \%$ [18]. The likelihood of an individual physician or payer recognizing the impact of the 2014 AAP guidance is low because of the small number of preterm infants in individual practices or healthcare plans. Additionally, impact assessment will be particularly difficult given that there is not routine testing for RSV, even among preterm infants [11]. Findings from the present study predict that the 2014 AAP guidance will result in additional burden to the healthcare system and families of preterm infants.

\section{CONCLUSION}

Although the impact of the new guidance is difficult to quantify among the overall infant population, the impact may be significant for this small, high-risk population, which, after having incurred high costs for birth hospitalization [19], will potentially be at risk for additional adverse outcomes such as RSV hospitalization, intensive care admission, and mechanical ventilation.

\section{ACKNOWLEDGMENTS}

Sponsorship for this study and article processing charges were funded by AstraZeneca. Medical writing assistance was provided by Anny Wu, PharmD, and Candace Lundin, DVM, MS of Complete Healthcare Communications, Inc. (Chadds Ford, PA, USA) and funded by AstraZeneca. All named authors meet the International Committee of Medical Journal Editors (ICMJE) criteria for authorship for this manuscript, take responsibility for the integrity of the work as a whole, and have given final approval to the version to be published. The authors thank Diana Swanson, PhD (AstraZeneca) for assistance with manuscript preparation. All authors had full access to all of the data in this study and take complete responsibility for the integrity of the data and accuracy of the data analysis. 
Disclosures. K. McLaurin and Doris Makari are employees of AstraZeneca. Archana Chatterjee received funding from AstraZeneca in the form of travel expenses to present findings from this study at a national conference.

Compliance with ethics guidelines. This article does not contain any new studies with humans or animal subjects performed by any of the authors.

Open Access. This article is distributed under the terms of the Creative Commons Attribution-NonCommercial 4.0 International License (http://creativecommons.org/licenses/ by-nc/4.0/), which permits any noncommercial use, distribution, and reproduction in any medium, provided you give appropriate credit to the original author(s) and the source, provide a link to the Creative Commons license, and indicate if changes were made.

\section{REFERENCES}

1. Nair H, Nokes DJ, Gessner BD, et al. Global burden of acute lower respiratory infections due to respiratory syncytial virus in young children: a systematic review and meta-analysis. Lancet. 2010;375:1545-55.

2. Lanari M, Silvestri M, Rossi GA. Respiratory syncytial virus risk factors in late preterm infants. J Matern Fetal Neonatal Med. 2009;22(Suppl 3):102-7.

3. SYNAGIS $^{\circledR} \quad$ Palivizumab. Full prescribing information, MedImmune, Gaithersburg, MD; 2014.

4. Committee on Infectious Diseases and Bronchiolitis Guidelines Committee. Updated guidance for palivizumab prophylaxis among infants and young children at increased risk of hospitalization for respiratory syncytial virus infection. Pediatrics. 2014;134:415-20.

5. American Academy of Pediatrics. Policy statement-modified recommendations for use of palivizumab for prevention of respiratory syncytial virus infections. Pediatrics. 2009;124.
6. American Academy of Pediatrics. Respiratory syncytial virus. In: Pickering LK, Baker CJ, Kimberlin DW, Long SS, editors. Red Book: 2012 Report of the Committee on Infectious Diseases. 29th ed. Elk Grove Village: American Academy of Pediatrics; 2012. p. 609-17.

7. Boyce TG, DeVincenzo J, Piedra PA. Updated RSV guidelines misinterpret available data [Letter to the editor]. Pediatrics. 2014. Available at: http:// pediatrics.aappublications.org/content/134/2/415. abstract/reply\#pediatrics_el_65418. Accessed Oct $20,2015$.

8. Domachowske JB. The 2014 AAP COID update on palivizumab. Available at: http://www. pediatricnews.com/columnists/single-view/the-2014aap-coid-update-on-palivizumab/3c76ea3404b6ca72 57a56682320acdac.html. Accessed Apr 17, 2015.

9. Standards for developing trustworthy clinical practice guidelines (CPGs). Institute of Medicine. Available at: http://iom.nationalacademies.org/ / media/Files/Report\%20Files/2011/Clinical-PracticeGuidelines-We-Can-Trust/Clinical\%20Practice\%20 Guidelines\%202011\%20Insert.pdf. Accessed Jul 1, 2015.

10. Centers for Disease Control and Prevention. Natality public-use data 2007-2010, on CDC WONDER Online Database, 2012. Available at: http://wonder.cdc.gov/natality-current.html. Accessed Jan 3, 2013.

11. Ambrose CS, Anderson EJ, Simoes EA, et al. Respiratory syncytial virus disease in preterm infants in the United States born at 32-35 weeks gestation not receiving immunoprophylaxis. Pediatr Infect Dis J. 2014;33:576-82.

12. Blanken MO, Rovers MM, Molenaar JM, et al. Respiratory syncytial virus and recurrent wheeze in healthy preterm infants. $\mathrm{N}$ Engl J Med. 2013;368:1791-9.

13. Stevens TP, Sinkin RA, Hall CB, Maniscalco WM, McConnochie KM. Respiratory syncytial virus and premature infants born at 32 weeks' gestation or earlier: hospitalization and economic implications of prophylaxis. Arch Pediatr Adolesc Med. 2000;154:55-61.

14. The IMpact-RSV Study Group. Palivizumab, a humanized respiratory syncytial virus monoclonal antibody, reduces hospitalization from respiratory syncytial virus infection in high-risk infants. Pediatrics. 1998;102:531-7.

15. Krilov LR, Halasa N, Thomas NJ, et al. SENTINEL1: An observational study of respiratory syncytial virus hospitalizations among United States infants born at 29-35 weeks' gestational age not receiving 
immunoprophylaxis [poster]. Presented at: National Medical Association. Detroit, MI; 2015.

16. Forbes ML, Hall CB, Jackson A, Masaquel AS, Mahadevia PJ. Comparative costs of hospitalisation among infants at high risk for respiratory syncytial virus lower respiratory tract infection during the first year of life. J Med Econ. 2010;13:136-41.

17. Horn SD, Smout RJ. Effect of prematurity on respiratory syncytial virus hospital resource use and outcomes. J Pediatr. 2003;143:S133-41.
18. Ambrose CS, Caspard H, Rizzo C, Stepka EC, Keenan G. Standard methods based on last menstrual period dates misclassify and overestimate US preterm births. J Perinatol. 2015 (Epub ahead of print).

19. Special care nursery admissions. Available at: https://www.marchofdimes.org/peristats/pdfdocs/ nicu_summary_final.pdf. Accessed Feb 6, 2015. 